\title{
CAEP/ACMU 2017 Research Abstract Awards
}

First place, Plenary Presentation, Grant Innes Research Paper and Presentation Award Ian Stiell

PL01 Creation of the Canadian heart failure risk scale for acute heart failure patients

\section{Second place, Plenary Presentation}

Audrey-Anne Brousseau

PL02 Derivation and validation of a feasible emergency department specific frailty index to predict adverse outcomes
Third place, Plenary Presentation

Christian Vaillancourt

PL03 Implementation of the Canadian C-Spine Rule by paramedics: a safety evaluation

Fourth place, Plenary Presentation, Top Resident Abstract Award Shannon Fernando

PL04 Initial Serum Lactate Predicts Deterioration in Emergency Department Patients with Sepsis
Top New Investigator Award

Leila Salehi

LO54 A descriptive analysis of ED length of stay of admitted patients 'boarded' in the emergency department

\section{Top Pediatric Abstract Award}

Amy Plint

LO25 How safe are our pediatric emergency departments? A multicentre, prospective cohort study

\section{Top Medical Student Project Award}

Dana Stewart

LO32 Are EMS offload delay patients at increased risk of adverse outcomes?

\section{Top Education Innovation Abstract Award}

Paula Sneath

LO13 GridlockED: an emergency medicine game and teaching tool

\section{CAEP-CanVECTOR Awards}

Sameer Sharif

LO81 Optimizing the use of CT scanning for pulmonary embolism in the emergency department
Eddy Lang

LO85 Substantial variation in CTPE ordering patterns and diagnostic yield in a large group of specialty-trained emergency physicians 


\section{CAEP Resident Research Abstract Awards}

Allison McConnell

LO33 Prehospital adverse events associated with nitroglycerin use in STEMI patients with right ventricle infarction

Luke Taylor

LO43 Does point of care ultrasound improve resuscitation markers in emergency department patients with undifferentiated hypotension? The first Sonography in Hypotension and Cardiac Arrest in the Emergency Department (SHOC-ED 1) Study; an international randomized controlled trial

\section{Sameer Masood}

LO66 Did the Choosing Wisely Canada campaign work? A retrospective analysis of its impact on emergency department imaging utilization for head injuries
Michael Beyea

LO68 Extracorporeal membrane oxygenation in the emergency department for resuscitation of out-of-hospital cardiac arrest patients: a systematic review

\section{Alexis Cournoyer}

LO71 For patients suffering from out-of-hospital cardiac arrest, is survival influenced by the capabilities of the receiving hospital?

Adam Harris

LO76 Emergency department procedural sedation in elderly patients

\section{Cristian Toarta}

LO096 Syncope prognosis based on emergency department diagnosis: a prospective cohort study

\section{Grizzly Den Presentations}

Khalifa Alqaydi

GD01 Age-adjusted D-dimer and two-site compression point-of-care ultrasonography to rule out acute deep vein thrombosis

Simon Berthelot

GD02 An international consensus study to identify quality indicators for ambulatory emergency care

\section{Naveen Poonai}

GD03 Hyoscine butylbromide (Buscopan) versus acetaminophen for non-surgical abdominal pain in children: a randomized controlled superiority trial

\section{Catherine Varner}

GD04 A blinded, randomized controlled trial of opioid analgesics for the management of acute fracture pain in older adults discharged from the emergency department

Kerstin de Wit

GD05 Careful Anticoagulation Review in Emergency Medicine (CARe-EM)

Justin Yan

GD06 Derivation and internal validation of a clinical prognostic tool for recurrent emergency visits for hyperglycemia in patients with diabetes mellitus: a multicentre prospective cohort study 


\section{CAEP/ACMU 2017 Grant Awards}

\section{CAEP-CanVECTOR Grant Awardee}

Kerstin de Wit

Patient preferences in emergency CT scanning for pulmonary embolism

\section{EMAF Grant Awardees}

Ariane Boutin

Randomized controlled trial evaluating the additive value of intranasal fentanyl on ibuprofen in the pain management of children with moderate to severe headaches

\section{Debra Eagles}

A study to evaluate barriers and facilitators to the emergency department discharge of patients with recent-onset atrial fibrillation and flutter

\section{Catherine Varner}

Challenging the dogma: A randomized controlled trial comparing prescribed light exercise to standard management for emergency department patients with acute mild traumatic brain injury

\section{Junior Investigator Awardees}

Shannon Fernando

Analysis of bystander CPR quality during out-ofhospital cardiac arrests using data derived from automated external defibrillators
Sarah Kilbertus

Transition to practice: evaluating the need for formal training in supervision and assessment techniques among senior emergency medicine residents and new to practice emergency physicians

Rohit Mohindra

A prospective randomized pilot trial to reduce readmission for frail elderly patients with acute decompensated heart failure

Trevor Skutezky

Improving patient care by engaging emergency department staff in a participatory design of a patient safety event reporting platform at an urban tertiary care hospital

\section{Robert Suttie}

Can emergency physicians reliably perform carotid artery point-of-care ultrasound to detect critical stenosis? 